\title{
Review of Arc Models in Distribution Networks
}

\author{
Qi Yin, Rui Ding \\ State Grid Deyang Power Supply Co. Ltd., Sichuan, 61800, China
}

\begin{abstract}
The incipient fault in underground cable is recognized as arc fault. Then the arc model selection is very important for the incipient fault detection. The arc features and some typical models have been introduced in detail, including traditional thermal based models, arc models in low voltage and models of arc in long free air. At last, the Kizilcay's model is recommended to analyze the incipient fault in underground cable for its accuracy and widely utilized. Finally, some conclusions are summarized.
\end{abstract}

\section{Introduction}

Arc is defined as a discharge of electricity through a gas, normally characterized by a voltage drop in the immediate vicinity of the cathode approximately equal to the ionization potential of the gas. It's a continuous luminous discharge of electricity across an insulating medium which is changed into a conducting medium due to a huge number of free electrons and ions. The arc is a complex entity, often mobile, always intensely hot and capable of many forms[1-4]. The arc is affected by many factors. Even under steady-state conditions, arc behaviour depends to a large degree on such factors as electrode material, nature and state of the gas medium, range of current magnitudes, and whether the arc is relatively long or relatively short. There are large number of tests have been done under different conditions. The volt-ampere curves are always different under different test conditions.

Under dynamic conditions, arcs show important additional properties which depend to an essential degree on rates of current change. Often arc behavior is so interrelated with electrical properties of the connected circuit that it is just as responsive to circuit conditions as it is to the physical state of the arc's surroundings[5]. The different of free burning arcs with low currents and high currents are as follows[7]: At low currents, i.e. less than $30 \mathrm{~A}$ in air, the controlling physical process is natural convection. It is assumed that the arc is vertical and that the input electrical energy produces an arc plasma which is carried upwards by natural convection. At very high currents, arc properties are also determined largely by convection, but the convection is determined by the selfmagnetic field of the arc. The increase of pressure inside the arc is greatest at the cathode spot where the arc crosssection is least and it is this high pressure which induces the axial flow

\section{The Development of Arc Analysis}

Although the arc is harm to the system operation, it is also have been utilized in electric arc furnace, and electric arc welding for it can produce high temperature. In this report, the different types of arc models are introduced, especially the arc in the fault.

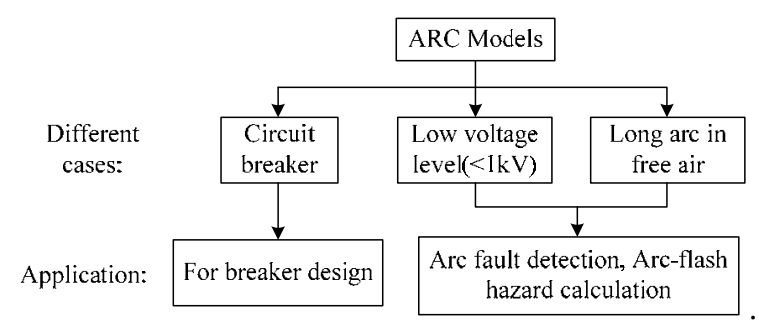

Figure 1. Different types of Arc model and its application.

The initial application of arc analysis is utilized in breaker design to extinguish the arc for it would damage the equipment. In recent three decades, the accurate arc models are developed for the arc fault detection. The accurate model is very important for the computer simulation to substitute large number of field tests which is high cost. And we can get the accurate features under different fault cases to verify the proposed methods.

The arc fault detection has been developed for many years. The arc in low voltage may cause fire hazard easily, so the arc detection is very important to prevent the fire disaster caused by arc. The transient faults in transmission system are always with arc in which the breaker should reclose as soon as possible to enhance the supply reliability. The incipient fault in power system is always the arc fault, then incipient fault detection became the arc fault detection. Also, the high impedance in medium-voltage distribution system always happen with arc, so the arc detection is very important to identify the high impedance fault.

\section{The Arc Models}

The arcs in power system have the similar nonlinear features which have been introduced in section 1, but the model is different with the different voltage level, 
medium, length and so on. Then the general characteristics of AC arc are introduced in 3.1. The instantaneous models in different conditions are introduced in 3.2. The arc models are different in different applications, including arc in breaker, low AC voltage system [8-22]. It is noted that the arc models for the energy calculation and secondary arc models are not introduced in this chapter.

\subsection{Steady Properties}

From the electrical properties of an arc under steady conditions (volt-ampere characteristic) point of view, a number of equations are derived from the experimental studies. The best known is that obtained by Ayrton.

$$
U=A+B L+\frac{C+D L}{I}
$$

where $\mathrm{U}$ is arc voltage, $\mathrm{I}$ is arc current and $\mathrm{L}$ is arc length, $\mathrm{A}$ is the anode/cathode voltage drop, B is the voltage gradient, $\mathrm{C}$ has the dimension of power, and $\mathrm{D}$ has the dimension of rate of power change over the arc length.

If in(1), L is made sufficiently large (the long arcs case), the terms involving parameters $\mathrm{A}$ and $\mathrm{C}$ may be neglected, and the characteristic equation becomes approximately

$$
U=\left(B+\frac{D}{I}\right) L
$$

If in (2) current is sufficiently large (the high current long arcs case), the arc voltage becomes a function only of the arc length, according to the following equation:

$$
U=B L
$$

Here, parameter $B$ represents the voltage gradient in the arc column. It is almost independent of arc current, so the long high current arc voltages are essentially determined by the arc length $\mathrm{L}$.

\subsection{Dynamic equation}

The basic assumption upon which mathematical "model" of the arc column are based on that the column conductance per unit length can be expressed as a prescribed function of the additional stored energy per unit length (often called heat content), Q, associated with the conducting state of the arc column:

$$
g=\frac{1}{R}=F(Q)=F\left[\int(W-N) d t\right]
$$

Where

$\mathrm{g}$ and $\mathrm{R}$ is conductance and resistance per unit length,

$\mathrm{Q}$ is excess energy content per unit length,

W is electric power input, watts per unit length,

$\mathrm{N}$ is rate of energy loss, watts per unit length, and

$\mathrm{t}$ is time.

In differential form, this equation is

$$
\frac{d g}{d t}=\frac{d}{d t}\left(\frac{1}{R}\right)=(W-N) \frac{\partial F(Q)}{\partial Q}
$$

Particular arc model equation depend upon the forms assumed for $\mathrm{F}(\mathrm{Q})$ and $\mathrm{N}$.

\subsubsection{Mayr's equation}

The equation proposed and used by Mayr assumes:

$$
F(Q)=K \exp \left(Q / Q_{0}\right)
$$

Where

$\mathrm{K}$ is an unspecified constant,

$\mathrm{Q}_{0}$ is a constant energy quantity,

$\mathrm{N}_{0}$ is a constant rate of energy loss, all per unit length of arc column.

This quantity, $\mathrm{Q}_{0}$, combined with the rate of energy loss, N0, determines the characteristic response frequency, $\mathrm{f}_{0}$, of the arc, or inversely the arc's time constant, which may be given the symbol $\theta$ and conveniently defined by relation

$$
\theta=\frac{Q_{0}}{N_{0}}
$$

Substitute of these three relations in the general equation (5) yields Mayr's arc model differential equation

$$
R \frac{d}{d t}\left(\frac{1}{R}\right)=\frac{1}{\theta}\left(\frac{W}{N_{0}}-1\right)
$$

If $\mathrm{W}$ is expressed as $\mathrm{I}^{2} \mathrm{R}$, this becomes

$$
\frac{d}{d t}\left(\frac{1}{R}\right)+\frac{1}{\theta}\left(\frac{1}{R}\right)=\frac{I^{2}}{\theta N_{0}}
$$

Which is a linear first order equation if the current $\mathrm{I}=\mathrm{I}(\mathrm{t})$ is determined solely by the connected circuit. If, on the other hand, $\mathrm{W}$ is written as $\mathrm{E}^{2 /} \mathrm{R}$, where $\mathrm{E}$ is the column voltage gradient, equation (8) becomes

$$
\frac{d R}{d t}-\frac{R}{\theta}=-\frac{E^{2}}{\theta N_{0}}
$$

which is a linear first order equation in $\mathrm{R}$ if the arc column gradient $\mathrm{E}=\mathrm{E}(\mathrm{t})$, is determined by the connected circuit alone.

\subsubsection{Cassie's equation}

A second arc model assume a column conductance simply proportional to $\mathrm{Q}$, expressible by the relation

$$
\frac{1}{R}=\frac{Q}{\theta E_{0}^{2}}
$$

And a power loss per unit length also proportional to $\mathrm{Q}$ according to

$$
N=\frac{Q}{\theta}
$$

where E0 is a characteristic voltage gradient. Substituting these relations in the basic equation (5) yield Cassie's differential equation which can be written

$$
R \frac{d}{d t}\left(\frac{1}{R}\right)=\frac{1}{\theta}\left[\left(\frac{E}{E_{0}}\right)^{2}-1\right]
$$

The linear first order equations may be obtained from (13) for cases where the circuit is an "infinitely stiff" source of either current or voltage. For impressed current, the equation can be written more conveniently 


$$
\frac{d}{d t}\left(\frac{1}{R^{2}}\right)+\frac{2}{\theta}\left(\frac{1}{R^{2}}\right)=\frac{2}{\theta}\left(\frac{I}{E_{0}}\right)^{2}
$$

which is linear in $1 / \mathrm{R}^{2}$. For impressed voltage, the equation may also be slightly simplified to

$$
\frac{d R}{d t}=\frac{R}{\theta}\left(1-\frac{E^{2}}{E_{0}^{2}}\right)
$$

which is linear in $\mathrm{R}$.

\section{Arc models in low-voltage AC system}

Arcing faults have been recognized as a potential hazard in low-voltage systems as far back as the 1920s, but the number of arcing-fault events began to rise in the 1960s as electrical systems changed to meet greater load demands [8]. There are many models are used for incident energy calculation, but only several models could be utilized for instantaneous value prediction which have been summarized in [9].

\subsection{Matthews model}

An arcing fault in a complex circuit can be described by the following differential equation:

$$
V_{\max } \sin (\omega t)=R \cdot i_{a r c}+L \frac{d i_{a r c}}{d t}+V_{a r c}
$$

The general solution for the arc current is

$$
i_{a r c}(t)=\frac{V_{\max }}{|Z|} \cdot\left[\sin (\omega t-\phi)-\frac{m}{\cos \phi}+\left(\frac{m}{\cos \phi}-\sin \left(\omega t_{a}-\phi\right)\right) \varepsilon^{\frac{-\omega\left(t-t_{a}\right)}{\tan \phi}}\right]
$$

The conduction angle, $\gamma$, can be determined with the FIND function in Mathcad from the following expression:

$$
\varepsilon^{-\gamma / \tan \phi}=\frac{\frac{m}{\cos \phi}-\sin \left(\omega t_{a}+\gamma-\phi\right)}{\frac{m}{\cos \phi}-\sin \left(\omega t_{a}-\phi\right)}
$$

In the first half-cycle, the arc current begins to flow at ta, when the supply voltage equals the restrike voltage. The current continues to flow until the conduction angle has been reached. At this point, the current becomes zero and the stored magnetic energy has been dissipated. The arc current in the second half cycle is a mirror image of the current in the first half-cycle.

\subsection{Stokes and Oppenlander model}

The models are expressed as differential equation, the arc currents are solved using a Runge-Kutta numerical method. It incorporates a current-dependent arc voltage based on an equation.

$$
V_{S}=R i_{\text {arc }}+L \frac{d i_{\text {arc }}}{d t}+(20+534 \cdot g) i_{\text {arc }}^{0.12}
$$

The arc voltage in (19) was derived from the pseudo constant portion of an ac signal, i.e., near the peak, and was determined from over 200 digitally sampled arc tests with more than two million arc current and voltage data points. The model was verified with the conditions - arc gaps from 5 to $500 \mathrm{~mm}$, arc current from 0.1 to $20000 \mathrm{~A}$ and arc powers from $50 \mathrm{~W}$ to over $30 \mathrm{MW}$.

\subsection{Lawrence's model}

The Lawrence's model incorporates an arc voltage based on the product of arc current and Fisher's equation for arc resistance.

$$
V_{S}=R i_{\text {arc }}+L \frac{d i_{\text {arc }}}{d t}+(25 \sqrt{39.37 \cdot g}) i_{\text {arc }}^{0.15}
$$

The gap is expressed in meters in (20), the arc voltage term incorporated in (20) was used in Fisher's method to directly determine the rms arc current, without solving for the instantaneous current values.

It is believed that this equation is approximately correct for 120 to $600 \mathrm{volt}, 60 \mathrm{~Hz}$ AC stable arcing burn down in a panel board or switchboard in which the arc length is approximately 2.5 inches.

\subsection{Others}

It is noted that there are many other models, such as the model proposed in [23] which new model derived by probability method according to the characteristics of three-phase fault arcs. Ref. [24] proposed a simplified model allows analyzing and determining basic parameters such as conduction angle, peak value, reduction factor of arc current, and power and energy supporting the understanding of the phenomenon of the arc flash.

\section{Arc Models in Breaker}

Breaker arc models can be classified into three categories [10]. Thermal and dielectric recovery models that describe the arc dynamical behaviour considering the impact of different interrupter parameters such as nozzle size and geometry, type of quenching medium and speed of flow, pressure, etc.. The second type of models is based on empirical forms. Both categories are efficient in determining the internal dimensions and quenching medium parameters of the interrupter. The third category of models is concerned with the arc external characteristics such as Cassie and Mary which have been introduced in 3.2. Although there are some modified method have been proposed for the analysis [11][12], they are not suit for the fault analysis. Then the arc models are not introduced in detail.

\section{Summary}

There are many types of arc models have been proposed for different destinations under different fault cases. It can be found that the model proposed by Kizilcay is the most widely used for arc fault analysis. The Kizilcay's model is time dependent dynamic resistance representation with emphasis of an empirical approach. It is flexible and is suit for different cases by changing the parameters in the model. However, the main problem of the model is the parameters selection: the 
unknown parameters must be estimated from test data or from experience.

\section{References}

[1] Stokes A D, Oppenlander W T. Electric arcs in open air[J]. Journal of Physics D: Applied Physics, 1991, 24(1): 26.

[2] Goda, Yutaka, et al. "Arc voltage characteristics of high current fault arcs in long gaps."Power Delivery, IEEE Transactions on 15.2 (2000): 791-795.

[3] Terzija, Vladimir, et al. "New Static "AirArc" EMTP Model of Long Arc in Free Air."Power Delivery, IEEE Transactions on26.3 (2011): 13441353.

[4] Elkalashy, Nagy I., et al. "Modeling and experimental verification of high impedance arcing fault in medium voltage networks."Dielectrics and Electrical Insulation, IEEE Transactions on 14.2 (2007): 375-383.

[5] Browne T E. The electric arc as a circuit element[J]. Journal of the Electrochemical Society, 1955, 102(1): 27-37.

[6] Fisher, Lawrence E. "Resistance of low-voltage ac arcs."Industry and General Applications, IEEE Transactions on 6 (1970): 607-616.

[7] Lowke J J. Simple theory of free-burning arcs[J]. Journal of physics D: Applied physics, 1979, 12(11): 1873.

[8] Gammon, Tammy, and John Matthews. "The historical evolution of arcing-fault models for lowvoltage systems." Industrial \&amp; Commercial Power Systems Technical Conference, 1999 IEEE.. IEEE, 1999.

[9] Gammon, Tammy, and John Matthews. "Instantaneous arcing-fault models developed for building system analysis."Industry Applications, IEEE Transactions on37.1 (2001): 197-203.

[10] Darwish, Hatem A., and Nagy I. Elkalashy. "Universal arc representation using EMTP."Power Delivery, IEEE Transactions on20.2 (2005): 772779.

[11] Tseng, King-Jet, Yaoming Wang, and D. Mahinda Vilathgamuwa. "An experimentally verified hybrid Cassie-Mayr electric arc model for power electronics simulations."Power Electronics, IEEE Transactions on12.3 (1997): 429-436.

[12] Schavemaker P H, Van der Slui L. An improved Mayr-type arc model based on current-zero measurements [circuit breakers][J]. Power Delivery, IEEE Transactions on, 2000, 15(2): 580-584.

[13] Terzija, Vladimir V., and H-J. Koglin. "On the modeling of long arc in still air and arc resistance calculation."Power Delivery, IEEE Transactions on19.3 (2004): 1012-1017.

[14] Djuric, M. B., and V. V. Terzija. "A new approach to the arcing faults detection for fast autoreclosure in transmission systems."Power Delivery, IEEE Transactions on10.4 (1995): 1793-1798.

[15] Kizilcay, M., and T. Pniok. "Digital simulation of fault arcs in power systems."European Transactions on Electrical Power1.1 (1991): 55-60.

[16] Kizilcay, Mustafa, and Piergiovanni La Seta. "Digital simulation of fault arcs in medium-voltage distribution networks."15th PSCC, Liege(2005): 2226.

[17] Kizilcay, M., and K - H. Koch. "Numerical fault arc simulation based on power arc tests."European Transactions on Electrical Power4.3 (1994): 177 185.

[18] Funabashi, Toshihisa, et al. "Influence of fault arc characteristics on the accuracy of digital fault locators."Power Delivery, IEEE Transactions on16.2 (2001): 195-199.

[19] Idarraga Ospina, G., D. Cubillos, and L. Ibanez. "Analysis of arcing fault models."Transmission and Distribution Conference and Exposition: Latin America, 2008 IEEE/PES. IEEE, 2008.

[20] $\mathrm{Xu}$, Zhihan.Fault location and incipient fault detection in distribution cables. Diss. The University of Western Ontario, 2011

[21] Elkalashy, Nagy Ibrahim.Modeling and detection of high impedance arcing fault in medium voltage networks. Helsinki University of Technology, 2007.

[22] Johns, A. T., R. K. Aggarwal, and Y. H. Song. "Improved techniques for modelling fault arcs an faulted EHV transmission systems." IEE Proceedings-Generation, Transmission and Distribution141.2 (1994): 148-154.

[23] $\mathrm{Wu}$, Huaren, et al. "Arc fault model for low-voltage AC systems."Power Delivery, IEEE Transactions on 20.2 (2005): 1204-1205.

[24] Parise, Giuseppe, Luigi Martirano, and Marco Laurini. "Simplified arc-fault model: the reduction factor of the arc current."Industry Applications, IEEE Transactions on49.4 (2013): 1703-1710. 